\title{
GENERALIZED PARAFERMIONS OF ORTHOGONAL TYPE
}

\author{
THOMAS CREUTZIG, VLADIMIR KOVALCHUK, AND ANDREW R. LINSHAW
}

\begin{abstract}
There is an embedding of affine vertex algebras $V^{k}\left(\mathfrak{g l}_{n}\right) \hookrightarrow V^{k}\left(\mathfrak{s l}_{n+1}\right)$, and the $\operatorname{coset} \mathcal{C}^{k}(n)=\operatorname{Com}\left(V^{k}\left(\mathfrak{g l}_{n}\right), V^{k}\left(\mathfrak{s l}_{n+1}\right)\right)$ is a natural generalization of the parafermion algebra of $\mathfrak{s l}_{2}$. It was called the algebra of generalized parafermions by the third author and was shown to arise as a one-parameter quotient of the universal two-parameter $\mathcal{W}_{\infty}$-algebra of type $\mathcal{W}(2,3, \ldots)$. In this paper, we consider an analogous structure of orthogonal type, namely $\mathcal{D}^{k}(n)=\operatorname{Com}\left(V^{k}\left(\mathfrak{s o}_{2 n}\right), V^{k}\left(\mathfrak{s o}_{2 n+1}\right)\right)^{\mathbb{Z}_{2}}$. We realize this algebra as a one-parameter quotient of the two-parameter even spin $\mathcal{W}_{\infty}$-algebra of type $\mathcal{W}(2,4, \ldots)$, and we classify all coincidences between its simple quotient $\mathcal{D}_{k}(n)$ and the algebras $\mathcal{W}_{\ell}\left(\mathfrak{s o}_{2 m+1}\right)$ and $\mathcal{W}_{\ell}\left(\mathfrak{s o}_{2 m}\right)^{\mathbb{Z}_{2}}$. As a corollary, we show that for the admissible levels $k=-(2 n-2)+\frac{1}{2}(2 n+$ $2 m-1)$ for $\widehat{\mathfrak{s o}}_{2 n}$ the simple affine algebra $L_{k}\left(\mathfrak{s o}_{2 n}\right)$ embeds in $L_{k}\left(\mathfrak{s o}_{2 n+1}\right)$, and the coset is strongly rational. As a consequence, the category of ordinary modules of $L_{k}\left(\mathfrak{s o}_{2 n+1}\right)$ at such a level is a braided fusion category.
\end{abstract}

\section{INTRODUCTION}

For $n \geq 1$, the natural embedding of Lie algebras $\mathfrak{g l}_{n} \hookrightarrow \mathfrak{s l}_{n+1}$ defined by

$$
a \mapsto\left(\begin{array}{cc}
a & 0 \\
0 & -\operatorname{tr}(a)
\end{array}\right),
$$

induces a vertex algebra homomorphism

$$
V^{k}\left(\mathfrak{g l}_{n}\right) \hookrightarrow V^{k}\left(\mathfrak{s l}_{n+1}\right) .
$$

The coset vertex algebra

$$
\mathcal{C}^{k}(n)=\operatorname{Com}\left(V^{k}\left(\mathfrak{g l}_{n}\right), V^{k}\left(\mathfrak{s l}_{n+1}\right)\right)
$$

was called the algebra of generalized parafermions in [LIII]. The reason for this terminology is that for $n=1, \mathcal{C}^{k}(1)$ is isomorphic to the parafermion algebra $N^{k}\left(\mathfrak{s l}_{2}\right)=\operatorname{Com}\left(\mathcal{H}, \mathfrak{s l}_{2}\right)$, where $\mathcal{H}$ denotes the Heisenberg algebra corresponding to the Cartan subalgebra $\mathfrak{h} \subseteq \mathfrak{s l}_{2}$.

By Theorem 8.1 of [LIII], $\mathcal{C}^{k}(n)$ is of type $\mathcal{W}\left(2,3, \ldots, n^{2}+3 n+1\right)$, i.e., it has a minimal strong generating set consisting of one field in each weight $2,3, \ldots, n^{2}+3 n+1$. This generalizes the case $n=1$, which appears in [DLY]. When $k$ is a positive integer, (1.1) descends to a map of simple affine vertex algebras $L_{k}\left(\mathfrak{g l}_{n}\right) \hookrightarrow L_{k}\left(\mathfrak{s l}_{n+1}\right)$, and the coset $\operatorname{Com}\left(L_{k}\left(\mathfrak{g l}_{n}\right), L_{k}\left(\mathfrak{s l}_{n+1}\right)\right)$ coincides with the simple quotient $\mathcal{C}_{k}(n)$ of $\mathcal{C}^{k}(n)$. By Theorem 13.1 of [ACL], we have an isomorphism

$$
\mathcal{C}_{k}(n) \cong \mathcal{W}_{\ell}\left(\mathfrak{s l}_{k}\right), \quad \ell=-k+\frac{k+n}{k+n+1} .
$$

In particular, $\mathcal{C}_{k}(n)$ is strongly rational, that is, $C_{2}$-cofinite and rational. This generalizes the case $n=1$, which was proved earlier in [ALY].

T. C. is supported by NSERC Discovery Grant \#RES0048511.

A. L. is supported by Simons Foundation Grant 635650 and NSF Grant DMS-2001484. 
A useful perspective on $\mathcal{C}^{k}(n)$ is that these algebras all arise in a uniform way as quotients of the universal two-parameter $\mathcal{W}_{\infty}$-algebra $\mathcal{W}(c, \lambda)$ of type $\mathcal{W}(2,3, \ldots)$; see Theorem 8.2 of [LIII]. This realization gives a nice conceptual explanation for the isomorphisms appearing in (1.3). Each one-parameter quotient of $\mathcal{W}(c, \lambda)$ corresponds to an ideal in $\mathbb{C}[c, \lambda]$, or equivalently, a curve in the parameter space $\mathbb{C}^{2}$ called the truncation curve. The truncation curves for $\mathcal{W}^{\ell}\left(\mathfrak{s l}_{m}\right)$ and $\mathcal{C}^{k}(n)$ are given by Equations 7.8 and 8.4 of [LIII], and the above isomorphisms correspond to intersection points on these curves.

The algebras $\mathcal{C}^{k}(n)$ appear naturally as building blocks for affine vertex algebras of type $A$. It is convenient to replace $\mathcal{C}^{k}(n)$ with $\tilde{\mathcal{C}}^{k}(n)=\mathcal{H} \otimes \mathcal{C}^{k}(n)$, where $\mathcal{H}$ is a rank one Heisenberg vertex algebra. Then we have

$$
\operatorname{Com}\left(V^{k}\left(\mathfrak{g l}_{n-1}\right), V^{k}\left(\mathfrak{g l}_{n}\right)\right) \cong \tilde{\mathcal{C}}^{k}(n-1),
$$

so $V^{k}\left(\mathfrak{g l}_{n}\right)$ can be regarded as an extension of $V^{k}\left(\mathfrak{g l}_{n-1}\right) \otimes \tilde{\mathcal{C}}^{k}(n-1)$. Iterating this procedure, we see that $V^{k}\left(\mathfrak{g l}_{n}\right)$ is an extension of

$$
\mathcal{H} \otimes \tilde{\mathcal{C}}^{k}(1) \otimes \tilde{\mathcal{C}}^{k}(2) \otimes \cdots \otimes \tilde{\mathcal{C}}^{k}(n-1) .
$$

Note that if $k$ is a positive integer, the simple quotient $L_{k}\left(\mathfrak{g l}_{n}\right)$ is then an extension of

$$
\mathcal{H} \otimes \tilde{\mathcal{C}}_{k}(1) \otimes \tilde{\mathcal{C}}_{k}(2) \otimes \cdots \otimes \tilde{\mathcal{C}}_{k}(n-1) \cong \mathcal{W}_{\ell_{1}}\left(\mathfrak{g l}_{k}\right) \otimes \mathcal{W}_{\ell_{2}}\left(\mathfrak{g l}_{k}\right) \otimes \cdots \otimes \mathcal{W}_{\ell_{n}}\left(\mathfrak{g l}_{k}\right),
$$

where $\ell_{i}=-k+\frac{k+n-i}{k+n+1-i}$. In [ACL], this was regarded as a noncommutative analogue of the Gelfand-Tsetin subalgebra of $U\left(\mathfrak{g l}_{n}\right)$. Similarly, we may regard the subalgebra (1.4) as the universal version of this structure.

The algebras $\mathcal{C}^{k}(n)$ also appear as building blocks for various $\mathcal{W}$-(super)algebras. For example, an important conjecture of Ito [I] asserts that the principal $\mathcal{W}$-algebra $\mathcal{W}^{\ell}\left(\mathfrak{s l}_{n+1 \mid n}\right)$ has a coset realization as

$$
\operatorname{Com}\left(V^{k+1}\left(\mathfrak{g l}_{n}\right), V^{k}\left(\mathfrak{s l}_{n+1}\right) \otimes \mathcal{F}(2 n)\right),
$$

where $\mathcal{F}(2 n)$ denotes the rank $2 n$ free fermion algebra, and $(\ell+1)(k+n+1)=1$. Ito's conjecture was stated in this form in [CLII], and these algebras have the same strong generating type by Lemma 7.12 of [CLII]. In the case $n=1$, the conjecture clearly holds because both sides are isomorphic to the $N=2$ superconformal algebra. The first nontrivial case $n=2$ was proven in [GL]. It was also shown in [GL] that the coset (1.5) is naturally an extension of $\mathcal{W}^{r}\left(\mathfrak{g l}_{n}\right) \otimes \mathcal{C}^{k}(n)$ for $r=-n+\frac{n+k}{n+k+1}$. An important ingredient in the proof of Ito's conjecture will be to show that $\mathcal{W}^{\ell}\left(\mathfrak{s l}_{n+1 \mid n}\right)$ is indeed an extension of $\mathcal{W}^{r}\left(\mathfrak{g l}_{n}\right) \otimes \mathcal{C}^{k}(n)$. Note that $\mathcal{C}^{k}(n)$ is itself a subalgebra of a $\mathcal{W}$-superalgebra of $\mathfrak{s l}_{n+1 \mid n}$ corresponding to a small hook-type nilpotent element [CLIII].

Generalized parafermion algebras of orthogonal type. There are two different analogues of $\mathcal{C}^{k}(n)$ in the orthogonal setting. We have natural embeddings $\mathfrak{s o}_{2 n} \hookrightarrow \mathfrak{s o}_{2 n+1} \hookrightarrow \mathfrak{s o}_{2 n+2}$, which induce homomorphisms of affine vertex algebras

$$
V^{k}\left(\mathfrak{s o}_{2 n}\right) \hookrightarrow V^{k}\left(\mathfrak{s o}_{2 n+1}\right) \hookrightarrow V^{k}\left(\mathfrak{s o}_{2 n+2}\right) .
$$

The cosets $\operatorname{Com}\left(V^{k}\left(\mathfrak{s o}_{2 n}\right), V^{k}\left(\mathfrak{s o}_{2 n+1}\right)\right)$ and $\operatorname{Com}\left(V^{k}\left(\mathfrak{s o}_{2 n+1}\right), V^{k}\left(\mathfrak{s o}_{2 n+2}\right)\right)$ both have actions of $\mathbb{Z}_{2}$, and we define

$$
\mathcal{D}^{k}(n)=\operatorname{Com}\left(V^{k}\left(\mathfrak{s o}_{2 n}\right), V^{k}\left(\mathfrak{s o}_{2 n+1}\right)\right)^{\mathbb{Z}_{2}}, \quad \mathcal{E}^{k}(n)=\operatorname{Com}\left(V^{k}\left(\mathfrak{s o}_{2 n+1}\right), V^{k}\left(\mathfrak{s o}_{2 n+2}\right)\right)^{\mathbb{Z}_{2}} .
$$

Both these algebras arise as one-parameter quotients of the universal even spin $\mathcal{W}_{\infty}$ algebra $\mathcal{W}^{\text {ev }}(c, \lambda)$ constructed recently by Kanade and the third author in [KL]. Such 
quotients of $\mathcal{W}^{\mathrm{ev}}(c, \lambda)$ are in bijection with a family of ideals $I$ in the polynomial ring $\mathbb{C}[c, \lambda]$, or equivalently, the truncation curves $V(I) \subseteq \mathbb{C}^{2}$. The main result in this paper is the explicit description of the truncation curve for $\mathcal{D}^{k}(n)$ for all $n$; see Theorem 3.3 . The proof is based on the coset realization of principal $\mathcal{W}$-algebras of type $D$ and a certain level-rank duality appearing in [ACL], which implies that

$$
\mathcal{D}_{2 m}(n) \cong \mathcal{W}_{\ell}\left(\mathfrak{s o}_{2 m}\right)^{\mathbb{Z}_{2}}, \quad \ell=-(2 m-2)+\frac{2 m+2 n-2}{2 m+2 n-1} .
$$

Here $\mathcal{D}_{2 m}(n)$ denotes the simple quotient of $\mathcal{D}^{2 m}(n)$. This is analogous to the isomorphisms (1.3) in type $A$. Since a similar coset realization of type $B$ principal $\mathcal{W}$-algebras is not available, we are currently unable to obtain an explicit description of $\mathcal{E}^{k}(n)$, and in this paper we only study $\mathcal{D}^{k}(n)$.

As in type $A$, there is a similar description of affine vertex algebras of orthogonal type as extensions of Gelfand-Tsetlin type subalgebras. Clearly $V^{k}\left(\mathfrak{s o}_{2 n+2}\right)$ is an extension of

$$
\mathcal{H} \otimes \mathcal{D}^{k}(1) \otimes \mathcal{E}^{k}(1) \otimes \mathcal{D}^{k}(2) \otimes \mathcal{E}^{k}(2) \otimes \cdots \otimes \mathcal{D}^{k}(n-1) \otimes \mathcal{E}^{k}(n-1) \otimes \mathcal{D}^{k}(n) \otimes \mathcal{E}^{k}(n),
$$

and similarly, $V^{k}\left(\mathfrak{s o}_{2 n+1}\right)$ is an extension of

$$
\mathcal{H} \otimes \mathcal{D}^{k}(1) \otimes \mathcal{E}^{k}(1) \otimes \mathcal{D}^{k}(2) \otimes \mathcal{E}^{k}(2) \otimes \cdots \otimes \mathcal{D}^{k}(n-1) \otimes \mathcal{E}^{k}(n-1) \otimes \mathcal{D}^{k}(n) .
$$

Additionally, $\mathcal{D}^{k}(n)$ is a building block for various $\mathcal{W}$-(super)algebras. For example, consider the principal $\mathcal{W}$-superalgebra $\mathcal{W}^{\ell}\left(\mathfrak{o s p}_{2 n \mid 2 n}\right)$ where $(\ell+1)(k+2 n-1)=1$. Note that 1 and $2 n-1$ are the dual Coxeter numbers of $\mathfrak{o s p}_{2 n \mid 2 n}$ and $\mathfrak{s o}_{2 n+1}$, respectively. The free fermion algebra $\mathcal{F}(2 n)$ carries an action of $L_{1}\left(\mathfrak{s o}_{2 n}\right)$, and it is expected that

$$
\mathcal{W}^{\ell}\left(\mathfrak{o s p} \mathfrak{p}_{2 n \mid 2 n}\right) \cong \operatorname{Com}\left(V^{k+1}\left(\mathfrak{s o}_{2 n}\right), V^{k}\left(\mathfrak{s o}_{2 n+1}\right) \otimes \mathcal{F}(2 n)\right)
$$

This algebra appears in physics in the duality of $N=1$ superconformal field theories and higher spin supergravities [CHR, $\mathrm{CV}]$, and this conjecture appeared in this context. Note that central charges coincide. It is apparent that the coset appearing in (1.9) is an extension of $\mathcal{W}^{r}\left(\mathfrak{s o}_{2 n}\right) \otimes \mathcal{D}^{k}(n)$ where $r=-(2 n-2)+\frac{k+2 n-2}{k+2 n-1}$. As in the case of Ito's conjecture, an important step in the proof of (1.9) will be to show that $\mathcal{W}^{\ell}\left(\mathfrak{o s p}_{2 n \mid 2 n}\right)$ is also an extension of this structure.

Applications. The first application of our main result is to classify all isomorphisms between the simple quotient $\mathcal{D}_{k}(n)$ and the simple algebras $\mathcal{W}_{\ell}\left(\mathfrak{s o}_{2 m+1}\right)$ and $\mathcal{W}_{\ell}\left(\mathfrak{s o}_{2 m}\right)^{\mathbb{Z}_{2}}$. Using results of $[\mathrm{KL}]$, this can be achieved by finding the intersection points between the truncation curve for $\mathcal{D}^{k}(n)$, and the truncation curves for $\mathcal{W}^{\ell}\left(\mathfrak{s o}_{2 m+1}\right)$ and $\mathcal{W}^{\ell}\left(\mathfrak{s o}_{2 m}\right)^{\mathbb{Z}_{2}}$, respectively. In the type $A$ case, we find only one family of points where $\mathcal{C}_{k}(n)$ is isomorphic to a strongly rational $\mathcal{W}$-algebra of type $A$; these appear in (1.3). In the orthogonal setting, the situation is more interesting. In addition to the isomorphisms (1.8) when $k$ is a positive integer, we also find that for $k=-(2 n-2)+\frac{1}{2}(2 n+2 m-1)$, we have an embedding of simple affine vertex algebras $L_{k}\left(\mathfrak{s o}_{2 n}\right) \rightarrow L_{k}\left(\mathfrak{s o}_{2 n+1}\right)$, and an isomorphism

$$
\mathcal{D}_{k}(n)=\operatorname{Com}\left(L_{k}\left(\mathfrak{s o}_{2 n}\right), L_{k}\left(\mathfrak{s o}_{2 n+1}\right)\right)^{\mathbb{Z}_{2}} \cong \mathcal{W}_{\ell}\left(\mathfrak{s o}_{2 m+1}\right), \quad \ell=-(2 m-1)+\frac{2 m+2 n-1}{2 m+2 n+1} .
$$

Since $\ell$ is a nondegenerate admissible level for $\mathfrak{s o}_{2 m+1}, \mathcal{W}_{\ell}\left(\mathfrak{s o}_{2 m+1}\right)$ is strongly rational [ArI, ArII]. These are new examples of cosets of non-rational vertex algebras by admissible level affine vertex algebras, which are strongly rational. 
This coset is also closely related to level-rank duality. Recall that $2 n(2 m+1)$ free fermions carry an action of $L_{2 n}\left(\mathfrak{s o}_{2 m+1}\right) \otimes L_{2 m+1}\left(\mathfrak{s o}_{2 n}\right)$. The levels shifted by the respective dual Coxeter numbers are $2 n+2 m-1$ in both cases. Therefore $L_{k}\left(\mathfrak{s o}_{2 n+1}\right)$ is an extension of $L_{k}\left(\mathfrak{s o}_{2 n}\right) \otimes \mathcal{W}_{\ell}\left(\mathfrak{s o}_{2 m+1}\right)$, where $\ell=-(2 m-1)+\frac{2 m+2 n-1}{2 m+2 n+1}$, i.e., both levels $k$ and $\ell$ shifted by the respective dual Coxeter numbers are of the form $(2 m+2 n-1) / v$ for $v=2$ and $v=2+2 m+2 n-1$. In particular, the shifted levels have the same numerator as the original level-rank duality and the two denominators only differ by a multiple of the numerator. Note that under certain vertex tensor category assumptions the tensor product of two vertex algebras can be extended to a larger vertex algebra with a certain multiplicity freeness condition if and only if the two vertex algebras have subcategories that are braid-reversed equivalent, see [CKMII, Main Thm. 3] for the precise statement. Applied to our setting, this means that there are vertex algebra extensions of $L_{k}\left(\mathfrak{s o}_{2 n}\right)$ and $\mathcal{W}_{\ell}\left(\mathfrak{s o}_{2 m+1}\right)$ that have subcategories of modules that are braid-reversed equivalent.

The theory of vertex algebra extensions, especially [CKMII, Thm. 5.12], then implies that the category of ordinary modules of $L_{k}\left(\mathfrak{s o}_{2 n+1}\right)$ at level $k=-(2 n-2)+\frac{1}{2}(2 n+2 m-1)$ is fusion, i.e. a rigid braided semisimple tensor category. This proves special cases of Conjecture 1.1 of [CHY].

Finally, our rationality results for $\mathcal{D}_{k}(n)$ suggest the existence of a new series of principal $\mathcal{W}$-superalgebras of $\mathfrak{o s p}_{2 n \mid 2 n}$ which are strongly rational. By Corollary 14.2 of [ACL], the coset $\operatorname{Com}\left(L_{k+1}\left(\mathfrak{s o}_{2 n}\right), L_{k}\left(\mathfrak{s o}_{2 n+1}\right) \otimes \mathcal{F}(2 n)\right)$ is strongly rational when $k$ is a positive integer. In view of the conjectured isomorphism (1.9), this implies that for $k$ a positive integer and $\ell$ satisfying $(\ell+1)(k+2 n-1)=1, \mathcal{W}_{\ell}\left(\mathfrak{o s p}_{2 n \mid 2 n}\right)$ is strongly rational. Similarly, it follows from Corollary 1.1 of [CKMII] that for $k=-(2 n-2)+\frac{1}{2}(2 n+2 m-1)$ and $\ell$ satisfying $(\ell+1)(k+2 n-1)=1$, the coset $\operatorname{Com}\left(L_{k+1}\left(\mathfrak{s o}_{2 n}\right), L_{k}\left(\mathfrak{s o}_{2 n+1}\right) \otimes \mathcal{F}(2 n)\right)$ is again strongly rational. This motivates the following

Conjecture 1.1. For $k=-(2 n-2)+\frac{1}{2}(2 n+2 m-1)$ and $\ell$ satisfying $(\ell+1)(k+2 n-1)=1$, $\mathcal{W}_{\ell}\left(\mathfrak{o s p}_{2 n \mid 2 n}\right)$ is strongly rational.

The conjecture is true for the $N=2$ super Virasoro algebra, i.e. the case $n=1$ [Ad]. Otherwise strong rationality for principal $\mathcal{W}$-superalgebras of orthosymplectic type is completely open. There is, however, a $C_{2}$-cofiniteness results in the case of $\mathfrak{o s p}_{2 \mid 2 n}$ [CGN], Cor. 5.19].

\section{VERTEX ALGEBRAS}

We shall assume that the reader is familiar with vertex algebras, and we use the same notation and terminology as the papers [LIII, KL]. We first recall the universal twoparameter vertex algebra $\mathcal{W}^{\text {ev }}(c, \lambda)$ of type $\mathcal{W}(2,4, \ldots)$, which was recently constructed in $[\mathrm{KL}]$. It is defined over the polynomial ring $\mathbb{C}[c, \lambda]$ and is generated by a Virasoro field $L$ of central charge $c$, and a weight 4 primary field $W^{4}$, and is strongly generated by fields $\left\{L, W^{2 i} \mid i \geq 2\right\}$ where $W^{2 i}=W_{(1)}^{4} W^{2 i-2}$ for $i \geq 3$. The idea of the construction is as follows.

(1) All structure constants in the OPEs of $L(z) W^{2 i}(w)$ and $W^{2 j}(z) W^{2 k}(w)$ for $2 i \leq 12$ and $2 j+2 k \leq 14$, are uniquely determined as elements of $\mathbb{C}[c, \lambda]$ by imposing the Jacobi identities among these fields. 
(2) This data uniquely and recursively determines all OPEs $L(z) W^{2 i}(w)$ and $W^{2 j}(z) W^{2 k}(w)$ over the ring $\mathbb{C}[c, \lambda]$ if a certain subset of Jacobi identities are imposed.

(3) By showing that the algebras $\mathcal{W}^{k}\left(\mathfrak{s p}_{2 m}\right)$ all arise as one-parameter quotients of $\mathcal{W}^{\text {ev }}(c, \lambda)$ after a suitable localization, we show that all Jacobi identities hold. Equivalently, $\mathcal{W}^{\text {ev }}(c, \lambda)$ is freely generated by the fields $\left\{L, W^{2 i} \mid i \geq 2\right\}$, and is the universal enveloping algebra of the corresponding nonlinear Lie conformal algebra [DSK].

$\mathcal{W}^{\mathrm{ev}}(c, \lambda)$ is simple as a vertex algebra over $\mathbb{C}[c, \lambda]$, but there is a certain discrete family of prime ideals $I=(p(c, \lambda)) \subseteq \mathbb{C}[c, \lambda]$ for which the quotient

$$
\mathcal{W}^{\mathrm{ev}, I}(c, \lambda)=\mathcal{W}^{\mathrm{ev}}(c, \lambda) / I \cdot \mathcal{W}^{\mathrm{ev}}(c, \lambda),
$$

is not simple as a vertex algebra over the ring $\mathbb{C}[c, \lambda] / I$. We denote by $\mathcal{W}_{I}^{\text {ev }}(c, \lambda)$ the simple quotient of $\mathcal{W}^{\text {ev }, I}(c, \lambda)$ by its maximal proper graded ideal $\mathcal{I}$. After a suitable localization, all one-parameter vertex algebras of type $\mathcal{W}(2,4, \ldots, 2 N)$ for some $N$ satisfying some mild hypotheses, can be obtained as quotients of $\mathcal{W}^{\mathrm{ev}}(c, \lambda)$ in this way. This includes the principal $\mathcal{W}$-algebras $\mathcal{W}^{k}\left(\mathfrak{s o}_{2 m+1}\right)$ and the orbifolds $\mathcal{W}^{k}\left(\mathfrak{s o}_{2 m}\right)^{\mathbb{Z}_{2}}$. The generators $p(c, \lambda)$ for such ideals arise as irreducible factors of Shapovalov determinants, and are in bijection with such one-parameter vertex algebras.

We also consider $\mathcal{W}^{\text {ev }, I}(c, \lambda)$ for maximal ideals

$$
I=\left(c-c_{0}, \lambda-\lambda_{0}\right), \quad c_{0}, \lambda_{0} \in \mathbb{C} .
$$

Then $\mathcal{W}^{\text {ev }, I}(c, \lambda)$ and its quotients are vertex algebras over $\mathbb{C}$. Given maximal ideals $I_{0}=\left(c-c_{0}, \lambda-\lambda_{0}\right)$ and $I_{1}=\left(c-c_{1}, \lambda-\lambda_{1}\right)$, let $\mathcal{W}_{0}$ and $\mathcal{W}_{1}$ be the simple quotients of $\mathcal{W}^{\mathrm{ev}, I_{0}}(c, \lambda)$ and $\mathcal{W}^{\mathrm{ev}, I_{1}}(c, \lambda)$. Theorem 8.1 of [KL] gives a simple criterion for $\mathcal{W}_{0}$ and $\mathcal{W}_{1}$ to be isomorphic. Aside from a few degenerate cases, we must have $c_{0}=c_{1}$ and $\lambda_{0}=\lambda_{1}$. This implies that aside from the degenerate cases, all other coincidences among the simple quotients of one-parameter vertex algebras $\mathcal{W}^{\mathrm{ev}, I}(c, \lambda)$ and $\mathcal{W}^{\mathrm{ev}, J}(c, \lambda)$, correspond to intersection points of their truncation curves $V(I)$ and $V(J)$.

We shall need the following result which is analogous to Theorem 6.2 of [LIII].

Theorem 2.1. Let $\mathcal{W}$ be a vertex algebra of type $\mathcal{W}(2,4, \ldots, 2 N)$ which is defined over some localization $R$ of $\mathbb{C}[c, \lambda] / I$, for some prime ideal I. Suppose that $\mathcal{W}$ is generated by the Virasoro field $L$ and a weight 4 primary field $W^{4}$. If in addition, the graded character of $\mathcal{W}$ agrees with that of $\mathcal{W}^{\text {ev }}(c, \lambda)$ up to weight 13 , then $\mathcal{W}$ is a quotient of $\mathcal{W}^{I}(c, \lambda)$ after localization.

Proof. First, note that Theorem 3.10 of [KL] holds without the simplicity assumption; see Remark 5.1 of [LIII] for a similar statement in the case of the algebra $\mathcal{W}(c, \lambda)$ of type $\mathcal{W}(2,3, \ldots)$. By Theorem 3.10 of $[\mathrm{KL}]$, it suffices to prove that the OPEs $L(z) W^{2 i}(w)$ and $W^{2 j}(z) W^{2 k}(w)$ for $2 i \leq 12$ and $2 j+2 k \leq 14$ in $\mathcal{W}$ are the same as the corresponding OPEs in $\mathcal{W}^{\mathrm{ev}}(c, \lambda)$ if the structure constants are replaced with their images in $R$. In this notation, $W^{2 i}=W_{(1)}^{4} W^{2 i-2}$ for $i \geq 3$. But this is automatic because the graded character assumption implies that there are no null vectors of weight $w \leq 13$ in the (possibly degenerate) nonlinear conformal algebra corresponding to $\left\{L, W^{2 i} \mid 2 \leq i \leq N\right\}$. 


\section{GENERALIZED PARAFERMIONS OF ORTHOGONAL TYPE}

For $n \geq 1$, the natural embedding $\mathfrak{s o}_{2 n} \hookrightarrow \mathfrak{s o}_{2 n+1}$ induces a vertex algebra homomorphism

$$
V^{k}\left(\mathfrak{s o}_{2 n}\right) \rightarrow V^{k}\left(\mathfrak{s o}_{2 n+1}\right) .
$$

The action of $\mathfrak{s o}_{2 n}$ on $V^{k}\left(\mathfrak{s o}_{2 n+1}\right)$ given by the zero modes of the generating fields integrates to an action of the orthogonal group $\mathrm{O}_{2 n}$. Therefore the coset

$$
\operatorname{Com}\left(V^{k}\left(\mathfrak{s o}_{2 n}\right), V^{k}\left(\mathfrak{s o}_{2 n+1}\right)\right)=V^{k}\left(\mathfrak{s o}_{2 n+1}\right)^{\mathfrak{s o}_{2 n}[t]}
$$

has a nontrivial action of $\mathbb{Z}_{2}$. We define

$$
\mathcal{D}^{k}(n)=\operatorname{Com}\left(V^{k}\left(\mathfrak{s o}_{2 n}\right), V^{k}\left(\mathfrak{s o}_{2 n+1}\right)\right)^{\mathbb{Z}_{2}} .
$$

It has Virasoro element $L^{\mathfrak{5 0} 2 n+1}-L^{\mathfrak{5 0} 2 n}$ with central charge

$$
c=\frac{k n(2 k+2 n-3)}{(k+2 n-2)(k+2 n-1)} .
$$

Note that in the case $n=1, \mathcal{D}^{k}(n) \cong N^{k}\left(\mathfrak{s l}_{2}\right)^{\mathbb{Z}_{2}}$ which is of type $\mathcal{W}(2,4,6,8,10)$ by Theorem 10.1 of $[\mathrm{KL}]$.

Lemma 3.1. For all $n \geq 1, \mathcal{D}^{k}(n)$ is of type $\mathcal{W}(2,4, \ldots, 2 N)$ for some $N$ satisfying $N \geq 2 n^{2}+3 n$. We conjecture, but do not prove, that $N=2 n^{2}+3 n$. Moreover, for generic values of $k, \mathcal{D}^{k}(n)$ is generated by the weight 4 primary field $W^{4}$.

Proof. By Theorem 6.10 of [CLII], we have

$$
\lim _{k \rightarrow \infty} \mathcal{D}^{k}(n) \cong \mathcal{H}(2 n)^{\mathrm{O}_{2 n}},
$$

and a strong generating set for $\mathcal{H}(2 n)^{\mathrm{O}_{2 n}}$ corresponds to a strong generating set for $\mathcal{D}^{k}(n)$ for generic values of $k$. Here $\mathcal{H}(2 n)$ denotes the rank $2 n$ Heisenberg vertex algebra. It was shown in [LII], Theorem 6.5, that $\mathcal{H}(2 n)^{\mathrm{O}_{2 n}}$ has the above strong generating type. By Lemma 4.2 of [LI], the weights 2 and 4 fields generate $\mathcal{H}(2 n)^{\mathrm{O}_{2 n}}$. In fact, it is easy to check that only the weight 4 field is needed, and that it can be replaced with a primary field which also generates the algebra. Finally, the statement that $\mathcal{D}^{k}(n)$ inherits these properties of $\mathcal{H}(2 n)^{\mathrm{O}_{2 n}}$ for generic values of $k$ is also clear; the argument is similar to the proof of Corollary 8.6 of [CLI].

Corollary 3.2. For all $n \geq 1$, there exists an ideal $K_{n} \subseteq \mathbb{C}[c, \lambda]$ and a localization $R_{n}$ of $\mathbb{C}[c, \lambda] / K_{n}$ such that $\mathcal{D}^{k}(n)$ is the simple quotient of $\mathcal{W}_{R_{n}}^{\mathrm{ev}, K_{n}}(c, \lambda)$.

Proof. This holds for $n=1$ by Theorem 10.1 of [KL]. For $n>1$, the simplicity of $\mathcal{D}^{k}(n)$ as a vertex algebra over a localization of $\mathbb{C}[k]$ follows from the simplicity of $\mathcal{H}(2 n)^{\mathrm{O}_{2 n}}$, which follows from [DLM]. In view of Theorems 2.1 and 3.1 , it then suffices to show that the graded characters of $\mathcal{D}^{k}(n)$ and $\mathcal{W}^{\mathrm{ev}}(c, \lambda)$ agree up to weight 13 . This follows from Weyl's second fundamental theorem of invariant theory for $\mathrm{O}_{2 n}[\mathrm{~W}]$, since there are no relations among the generators of weight less than $4 n^{2}+6 n+2$.

Theorem 3.3. For all $n \geq 2, \mathcal{D}^{k}(n)$ is isomorphic to a localization of the quotient $\mathcal{W}_{K_{n}}^{e v}(c, \lambda)$, where the ideal $K_{n} \subseteq \mathbb{C}[c, \lambda]$ is described explicitly via the parametrization $k \mapsto\left(c_{n}(k), \lambda_{n}(k)\right)$ 
given by

$$
\begin{aligned}
c_{n}(k)= & \frac{k n(2 k+2 n-3)}{(k+2 n-2)(k+2 n-1)}, \quad \lambda_{n}(k)=\frac{(k+2 n-2)(k+2 n-1) p_{n}(k)}{7(k-2)(k+n-1)(2 n-1) q_{n}(k) r_{n}(k)}, \\
p_{n}(k)= & -112+188 k-62 k^{2}-26 k^{3}+12 k^{4}+744 n-1336 k n+857 k^{2} n-252 k^{3} n \\
& +36 k^{4} n-1720 n^{2}+2534 k n^{2}-1198 k^{2} n^{2}+188 k^{3} n^{2}+1632 n^{3}-1544 k n^{3} \\
& +304 k^{2} n^{3}-544 n^{4}+152 k n^{4}, \\
q_{n}(k)= & 20-19 k+6 k^{2}-42 n+28 k n+28 n^{2}, \\
r_{n}(k)= & 44-66 k+22 k^{2}-132 n+73 k n+10 k^{2} n+88 n^{2}+10 k n^{2} .
\end{aligned}
$$

Proof. Let $n$ be fixed. In view of Corollary 3.2 and the fact that all structure constants in $\mathcal{D}^{k}(n)$ are rational functions of $k$, there is some rational function $\lambda_{n}(k)$ of $k$ such that $\mathcal{D}^{k}(n)$ is obtained from $\mathcal{W}^{\text {ev }}(c, \lambda)$ by setting $c=c_{n}(k)$ and $\lambda=\lambda_{n}(k)$, and then taking the simple quotient. It is not obvious yet that $\lambda_{n}(k)$ is a rational function of $n$ as well.

For $k$ a positive integer, it is well known [KW] that the map $V^{k}\left(\mathfrak{s o}_{2 n}\right) \rightarrow V^{k}\left(\mathfrak{s o}_{2 n+1}\right)$ descends to a homomorphism of simple algebras $L_{k}\left(\mathfrak{s o}_{2 n}\right) \rightarrow L_{k}\left(\mathfrak{s o}_{2 n+1}\right)$. Letting $\mathcal{D}_{k}(n)$ denote the simple quotient of $\mathcal{D}^{k}(n)$, it is apparent from Lemma 2.1 of [ACKL] and Theorem 8.1 of [CLII] that $\operatorname{Com}\left(L_{k}\left(\mathfrak{s o}_{2 n}\right), L_{k}\left(\mathfrak{s o}_{2 n+1}\right)\right)$ is simple and coincides with the simple quotient of $\operatorname{Com}\left(V^{k}\left(\mathfrak{s o}_{2 n}\right), V^{k}\left(\mathfrak{s o}_{2 n+1}\right)\right)$. Moreover, taking $\mathbb{Z}_{2}$-invariants preserves simplicity, hence

$$
\mathcal{D}_{k}(n) \cong \operatorname{Com}\left(L_{k}\left(\mathfrak{s o}_{2 n}\right), L_{k}\left(\mathfrak{s o}_{2 n+1}\right)\right)^{\mathbb{Z}_{2}} .
$$

Next, by Corollary 1.3 of [ACL], for all $n \geq 1$ and $m \geq 2$, we have an isomorphism

$$
\left(\left(L_{2 m}\left(\mathfrak{s o}_{2 n+1}\right) \oplus \mathbb{L}_{2 m}\left(2 m \omega_{1}\right)\right)^{\mathfrak{s o}_{2 n}[t]}\right)^{\mathbb{Z}_{2} \times \mathbb{Z}_{2}} \cong \mathcal{W}_{\ell}\left(\mathfrak{s o}_{2 m}\right), \quad \ell=-(2 m-2)+\frac{2 n+2 m-2}{2 n+2 m-1}
$$

In this notation, $\omega_{1}$ denotes the first fundamental weight of $\mathfrak{s o}_{2 n+1}$ and $\mathbb{L}_{2 m}\left(2 m \omega_{1}\right)$ denotes the simple quotient of the corresponding Weyl module.

Note that $\left(L_{2 m}\left(\mathfrak{s o}_{2 n+1}\right)^{\mathfrak{s o}_{2 n}[t]}\right)^{\mathbb{Z}_{2}}=\mathcal{D}_{2 m}(n)$ is manifestly a subalgebra of the left hand side of (3.4). Also, the lowest-weight component of $\mathbb{L}_{2 m}\left(2 m \omega_{1}\right)$ has conformal weight $m$. If $m>4$, the left-hand side then has a unique primary weight 4 field which lies in $\mathcal{D}_{2 m}(n)$. Similarly, since $\mathcal{W}_{\ell}\left(\mathfrak{s o}_{2 m}\right)$ has strong generators in weights $2,4, \ldots, 2 m$ and $m$, for $m>4$ the right hand side has a unique primary weight 4 field, which lies in the $\mathbb{Z}_{2}$-orbifold $\mathcal{W}_{\ell}\left(\mathfrak{s o}_{2 m}\right)^{\mathbb{Z}_{2}}$.

Since $\mathcal{D}^{k}(n)$ is generated by the weight 4 field as a one-parameter vertex algebra, the weight 4 field must generate $\mathcal{D}_{2 m}(n)$ for all $m$ sufficiently large. By Corollary 6.1 of [KL], $\mathcal{W}^{\ell}\left(\mathfrak{s o}_{2 m}\right)^{\mathbb{Z}_{2}}$ is generated by the weight 4 field as a one-parameter vertex algebra; equivalently, this holds for generic values of $\ell$. By the same argument as Proposition A.4 of [ALY], the vertex Poisson structure on the associated graded algebra gr $\mathcal{W}^{\ell}\left(\mathfrak{s o}_{2 m}\right)$ with respect to Li's canonical filtration, is independent of $\ell$ for all noncritical values of $\ell$. In particular this holds for the subalgebra $\left(\operatorname{gr} \mathcal{W}^{\ell}\left(\mathfrak{s o}_{2 m}\right)\right)^{\mathbb{Z}_{2}}=\operatorname{gr}\left(\mathcal{W}^{\ell}\left(\mathfrak{s o}_{2 m}\right)^{\mathbb{Z}_{2}}\right)$. It follows from the same argument as Proposition A.3 of [ALY] that $\mathcal{W}^{\ell}\left(\mathfrak{s o}_{2 m}\right)^{\mathbb{Z}_{2}}$ is generated by the weights 2 and 4 fields for all noncritical values of $\ell$, and the same therefore holds for the simple quotient $\mathcal{W}_{\ell}\left(\mathfrak{s o}_{2 m}\right)^{\mathbb{Z}_{2}}$. Finally, for $\ell=-(2 m-2)+\frac{2 n+2 m-2}{2 n+2 m-1}$, it is straightforward to verify that the Virasoro field can be generated from the weight 4 field, so the weight 4 field generates the whole algebra. 
Therefore if $m$ is sufficiently large, we obtain

$$
\mathcal{D}_{2 m}(n) \cong \mathcal{W}_{\ell}\left(\mathfrak{s o}_{2 m}\right)^{\mathbb{Z}_{2}}, \quad \ell=-(2 m-2)+\frac{2 m+2 n-2}{2 m+2 n-1} .
$$

In fact, we will see later (Theorem 4.1) that this holds for all $m \geq 2$.

Finally, the truncation curve that realizes $\mathcal{W}_{\ell}\left(\mathfrak{s o}_{2 m}\right)^{\mathbb{Z}_{2}}$ as a quotient of $\mathcal{W}^{\mathrm{ev}}(c, \lambda)$ is given by Theorem 6.3 of $[\overline{\mathrm{KL}}]$, and in parametric form by Equation (B.1) of [KL]. In view of (3.5), we must have $\lambda_{n}(2 m)=\lambda_{m}(\ell)$ for $\ell=-(2 m-2)+\frac{2 n+2 m-2}{2 n+2 m-1}$ for $m$ sufficiently large, where $\lambda_{m}(\ell)$ is given by Equation (B.1) of [KL]. If follows that for infinitely many values of $k, \lambda_{n}(k)$ is given by the above formula (3.3). Since $\lambda_{n}(k)$ is a rational function of $k$, this equality holds for all $k$ where it is defined. This completes the proof.

\section{COINCIDENCES}

In this section, we shall use Theorem 3.3 to classify all coincidences between the simple quotient $\mathcal{D}_{k}(n)$ and the $\mathbb{Z}_{2}$-orbifold $\mathcal{W}_{\ell}\left(\mathfrak{s o}_{2 m}\right)^{\mathbb{Z}_{2}}$, as well as $\mathcal{W}_{\ell}\left(\mathfrak{s o}_{2 m+1}\right)$. We also classify all coincidences between $\mathcal{D}_{k}(n)$ and $\mathcal{D}_{\ell}(m)$ for $m \neq n$.

Theorem 4.1. For $n \geq 1$ and $m \geq 2$, aside from the critical levels $k=-2 n+2$ and $k=$ $-2 n+1$, and the degenerate cases $c=\frac{1}{2},-24$, all isomorphisms $\mathcal{D}_{k}(n) \cong \mathcal{W}_{\ell}\left(\mathfrak{s o}_{2 m}\right)^{\mathbb{Z}_{2}}$ appear on the following list:

(1) $k=2 m, \quad \ell=-(2 m-2)+\frac{2 n+2 m-2}{2 n+2 m-1}$,

(2) $k=-(2 n-2)-\frac{2 n-1}{2(m-1)}, \quad \ell=-(2 m-2)+\frac{2 m-2 n-1}{2(m-1)}$,

(3) $k=-(2 n-2)+\frac{n-m}{m}, \quad \ell=-(2 m-2)+\frac{m-n}{m}$.

Proof. Recall first that $\mathcal{W}_{\ell}\left(\mathfrak{s o}_{2 m}\right)^{\mathbb{Z}_{2}}$ is realized as the simple quotient of $\mathcal{W}^{\text {ev }, J_{m}}(c, \lambda)$, where the ideal $J_{m} \subseteq \mathbb{C}[c, \lambda]$ is given in parametrized form by Equation (B.1) of [KL]. First, we exclude the values of $k$ and $\ell$ which are poles of the functions $\lambda_{n}(k)$ given by (3.3), and $\lambda_{m}(\ell)$ given by Equation (B.1) of [KL], since at these values, $\mathcal{D}^{k}(n)$ and $\mathcal{W}_{\ell}\left(\mathfrak{s o}_{2 m}\right)^{\mathbb{Z}_{2}}$ are not quotients of $\mathcal{W}^{\text {ev }}(c, \lambda)$. For all other noncritical values of $k$ and $\ell, \mathcal{D}^{k}(n)$ and $\mathcal{W}_{\ell}\left(\mathfrak{s o}_{2 m}\right)^{\mathbb{Z}_{2}}$ are obtained as quotients of $\mathcal{W}^{\mathrm{ev}, I_{n}}(c, \lambda)$ and $\mathcal{W}^{\mathrm{ev}, J_{m}}(c, \lambda)$, respectively. By Corollary 8.2 of $[\overline{\mathrm{KL}}]$, aside from the degenerate cases given by Theorem 8.1 of [ [KL], all other coincidences $\mathcal{D}_{k}(n) \cong \mathcal{W}_{\ell}\left(\mathfrak{s o}_{2 m}\right)^{\mathbb{Z}_{2}}$ correspond to intersection points on the truncation curves $V\left(K_{n}\right)$ and $V\left(J_{m}\right)$. A calculation shows that $V\left(K_{n}\right) \cap V\left(J_{m}\right)$ consists of exactly five points $(c, \lambda)$, namely,

$$
\begin{aligned}
& \left(-24,-\frac{1}{245}\right), \quad\left(\frac{1}{2},-\frac{2}{49}\right), \quad\left(\frac{m n(4 m+2 n-3)}{(m+n-1)(2 m+2 n-1)}, \lambda_{1}\right) \\
& \left(-\frac{2 m n(3-4 m-2 n+4 m n)}{2 m-2 n-1}, \lambda_{2}\right), \quad\left(-\frac{(2 m n+m-2 n)(2 m n-m-n)}{m-n}, \lambda_{3}\right) .
\end{aligned}
$$


Here

$$
\begin{aligned}
& \lambda_{1}=\frac{(m+n-1)(2 m+2 n-1) g}{7(m-1)(2 m+n-1)(2 n-1) g h}, \\
& f=-28+94 m-62 m^{2}-52 m^{3}+48 m^{4}+186 n-668 m n+857 m^{2} n-504 m^{3} n \\
& +144 m^{4} n-430 n^{2}+1267 m n^{2}-1198 m^{2} n^{2}+376 m^{3} n^{2}+408 n^{3}-772 m n^{3} \\
& +304 m^{2} n^{3}-136 n^{4}+76 m n^{4} \\
& g=10-19 m+12 m^{2}-21 n+28 m n+14 n^{2}, \\
& h=22-66 m+44 m^{2}-66 n+73 m n+20 m^{2} n+44 n^{2}+10 m n^{2} . \\
& \lambda_{2}=\frac{(1-2 m+2 n) f}{7(1-2 m+2 m n)(-1-2 n+4 m n) g h}, \\
& f=14-33 m-2 m^{2}+24 m^{3}+74 n-404 m n+873 m^{2} n-696 m^{3} n+144 m^{4} n \\
& +80 n^{2}-178 m n^{2}-260 m^{2} n^{2}+452 m^{3} n^{2}-112 m^{4} n^{2}-24 n^{3}+264 m n^{3} \\
& -348 m^{2} n^{3}+256 m^{3} n^{3}-64 m^{4} n^{3}+72 m n^{4}-128 m^{2} n^{4}-48 m^{3} n^{4}+32 m^{4} n^{4}, \\
& g=-10+19 m-12 m^{2}-2 n+22 m n-8 m^{2} n-12 n^{2}-8 m n^{2}+8 m^{2} n^{2}, \\
& h=11-22 m+22 n+15 m n-20 m^{2} n-10 m n^{2}+20 m^{2} n^{2} \text {. } \\
& \lambda_{3}=\frac{(n-m) f}{7(m-1)(2 n-1)(m-n+2 m n) g h}, \\
& f=-34 m^{3}+19 m^{4}+68 m^{2} n-38 m^{3} n-22 m n^{2}-185 m^{2} n^{2}+302 m^{3} n^{2}-80 m^{4} n^{2} \\
& -12 n^{3}+204 m n^{3}-302 m^{2} n^{3}+80 m^{3} n^{3}-36 n^{4}+100 m n^{4}-40 m^{2} n^{4}-40 m^{3} n^{4} \\
& +16 m^{4} n^{4} \\
& g=-7 m^{2}+7 m n-6 n^{2}-4 m n^{2}+4 m^{2} n^{2}, \\
& h=-22 m-5 m^{2}+22 n+5 m n+10 n^{2}-30 m n^{2}+20 m^{2} n^{2} .
\end{aligned}
$$

By Theorem 8.1 of [ $[\mathrm{KL}]$, the first two intersection points occur at degenerate values of $c$. By replacing the parameter $c$ with the levels $k$ and $\ell$, we see that the remaining intersection points yield the nontrivial isomorphisms in Theorem 4.1. Moreover, by Corollary 8.2 of $[\mathrm{KL}]$, these are the only such isomorphisms except possibly at the values of $k, \ell$ excluded above.

Finally, suppose that $k$ is a pole of the function $\lambda_{n}(k)$ given by (3.3). It is not difficult to check that the corresponding values of $\ell$ for which $c_{n}(k)=c_{m}(\ell)$, are not poles of $\lambda_{m}(\ell)$. As above, $c_{n}(k)$ and $\lambda_{n}(k)$ are given by (3.3), and $c_{m}(\ell)$ and $\lambda_{m}(\ell)$ are given by Equation (B.1) of $[\mathrm{KL}]$. It follows that there are no additional coincidences at the excluded points.

Next, we classify the coincidences between $\mathcal{D}_{k}(n)$ and $\mathcal{W}_{\ell}\left(\mathfrak{s o}_{2 m+1}\right)$.

Theorem 4.2. For $n \geq 1$ and $m \geq 2$, aside from the critical levels $k=-2 n+2$ and $k=-2 n+1$, and the degenerate cases $c=\frac{1}{2},-24$, all isomorphisms $\mathcal{D}_{k}(n) \cong \mathcal{W}_{\ell}\left(\mathfrak{s o}_{2 m+1}\right)$ appear on the following list:

$$
\text { (1) } k=-(2 n-2)+\frac{1}{2}(2 n+2 m-1), \quad \ell=-(2 m-1)+\frac{2 m+2 n-1}{2 m+2 n+1} \text {, }
$$


(2) $k=-(2 n-2)+\frac{2 n-2 m-1}{2 m+2}, \quad \ell=-(2 m-1)+\frac{2 m-2 n+1}{2 m+2}$,

(3) $k=-(2 n-2)-\frac{n}{m}, \quad \ell=-(2 m-1)+\frac{m-n}{m}$,

(4) $k=-(2 n-2)-\frac{2(n-1)}{2 m-1}, \quad \ell=-(2 m-1)+\frac{2 m-1}{2 m-2 n+1}$.

(5) $k=-(2 n-2)+\frac{2(n-m-1)}{2 m+1}, \quad \ell=-(2 m-1)+\frac{2 m+1}{2(m-n+1)}$.

Proof. The argument is the same as the proof of Theorem 4.1 . First, $\mathcal{W}_{\ell}\left(\mathfrak{s o}_{2 m+1}\right)$ is realized as the simple quotient of $\mathcal{W}^{\mathrm{ev}, I_{m}}(c, \lambda)$ where the ideal $I_{m} \subseteq \mathbb{C}[c, \lambda]$ is parametrized explicitly by Equation (A.3) of [KL]. The above isomorphisms all arise from the intersection points between the truncation curves $V\left(K_{n}\right)$ for $\mathcal{D}^{k}(n)$ and $V\left(I_{m}\right)$ for $\mathcal{W}_{\ell}\left(\mathfrak{s o}_{2 m+1}\right)$. A calculation shows that there are exactly 7 intersection points: the degenerate points $\left(\frac{1}{2},-\frac{2}{49}\right)$ and $\left(-24,-\frac{1}{245}\right)$, and the five nontrivial ones appearing above. One then has to rule out additional coincidences at the points where $\mathcal{D}_{k}(n)$ does not arise as a quotient of $\mathcal{W}^{\text {ev }}(c, \lambda)$, namely, the poles of $\lambda_{n}(k)$. The details are straightforward and are left to the reader.

Finally, we classify all isomorphisms $\mathcal{D}_{k}(m) \cong \mathcal{D}_{\ell}(n)$ for $n \neq m$.

Theorem 4.3. For $m, n \geq 1$ and $n \neq m$, aside from the degenerate cases $c=\frac{1}{2},-24$ and poles of $c_{n}(k), \lambda_{n}(k)$ and $c_{m}(k), \lambda_{m}(k)$ the complete list of isomorphisms $\mathcal{D}_{k}(m) \cong \mathcal{D}_{\ell}(n)$ is the following:

(1) $k=-(2 m-2)+\frac{2(m-1)}{1+2 n}, \quad \ell=-(2 n-2)-\frac{2 m+2 n-1}{2(m-1)}$,

(2) $k=-(2 m-2)-\frac{2 m+2 n-1}{2(n-1)}, \quad \ell=-(2 n-2)+\frac{2(n-1)}{1+2 m}$.

The proof is similar to the proof of Theorem 4.1 and is omitted.

\section{SOME RATIONAL COSETS}

By composing the map $V^{k}\left(\mathfrak{s o}_{2 n}\right) \rightarrow V^{k}\left(\mathfrak{s o}_{2 n+1}\right)$ with the quotient map $V^{k}\left(\mathfrak{s o}_{2 n+1}\right) \rightarrow$ $L_{k}\left(\mathfrak{s o}_{2 n+1}\right)$, we obtain an embedding

$$
\tilde{V}^{k}\left(\mathfrak{s o}_{2 n}\right) \hookrightarrow L_{k}\left(\mathfrak{s o}_{2 n+1}\right),
$$

where $\tilde{V}^{k}\left(\mathfrak{s o}_{2 n}\right)$ denotes the quotient of $V^{k}\left(\mathfrak{s o}_{2 n}\right)$ by the kernel $\mathcal{J}_{k}$ of the above composition. In general, it is a difficult and important problem to determine when $\mathcal{J}_{k}$ is the maximal proper graded ideal, or equivalently, when $\tilde{V}^{k}\left(\mathfrak{s o}_{2 n}\right)=L_{k}\left(\mathfrak{s o}_{2 n}\right)$. In the case where $k$ is an admissible level for $\widehat{\mathfrak{s o}_{2 n}}$, Lemma 2.1 of [ACKL] would then imply that $\operatorname{Com}\left(L_{k}\left(\mathfrak{s o}_{2 n}\right), L_{k}\left(\mathfrak{s o}_{2 n+1}\right)\right)$ is simple, and hence its orbifold $\operatorname{Com}\left(L_{k}\left(\mathfrak{s o}_{2 n}\right), L_{k}\left(\mathfrak{s o}_{2 n+1}\right)\right)^{\mathbb{Z}_{2}}$ would be simple as well [DLM]. Additionally, Theorem 8.1 of [CLII] would imply that $\operatorname{Com}\left(L_{k}\left(\mathfrak{s o}_{2 n}\right), L_{k}\left(\mathfrak{s o}_{2 n+1}\right)\right)^{\mathbb{Z}_{2}}$ coincides with the simple quotient $\mathcal{D}_{k}(n)$ of $\mathcal{D}^{k}(n)$. This is particularly interesting in the cases where $\mathcal{D}_{k}(n)$ is strongly rational.

We conclude by proving this for first family in Theorem 4.2. These are new examples of cosets of non-rational vertex algebras by admissible level affine vertex algebras, which are strongly rational. 
Lemma 5.1. For $n \geq 2$ and $m \geq 0$, we have an embedding of simple affine vertex algebras

$$
L_{k}\left(\mathfrak{s o}_{2 n}\right) \hookrightarrow L_{k}\left(\mathfrak{s o}_{2 n+1}\right), \quad k=-(2 n-2)+\frac{1}{2}(2 n+2 m-1) .
$$

Proof. We proceed by induction on $m$. In the case $m=0$, we have $k=-n+\frac{3}{2}$, and it is well known that there exists a conformal embedding $L_{k}\left(\mathfrak{s o}_{2 n}\right) \hookrightarrow L_{k}\left(\mathfrak{s o}_{2 n+1}\right)$, see e.g. Section 3 of [AKMPP]. Next, we assume the result for $m-1$, so that $k=-n+\frac{3}{2}+m-1$. Recall that the rank $2 n+1$ free fermion algebra $\mathcal{F}(2 n+1)$ admits an action of $L_{1}\left(\mathfrak{s o}_{2 n+1}\right)$, as well as an action of $L_{1}\left(\mathfrak{s o}_{2 n}\right)$ via the embedding $L_{1}\left(\mathfrak{s o}_{2 n}\right) \hookrightarrow L_{1}\left(\mathfrak{s o}_{2 n+1}\right)$. The image of $L_{1}\left(\mathfrak{s o}_{2 n}\right)$ lies in the subalgebra $\mathcal{F}(2 n) \subseteq \mathcal{F}(2 n+1)$.

Since $k$ is admissible for $\mathfrak{s o}_{2 n+1}$, it is known [KW] that we have a diagonal embedding of simple affine vertex algebras

$$
L_{k+1}\left(\mathfrak{s o}_{2 n+1}\right) \hookrightarrow L_{k}\left(\mathfrak{s o}_{2 n+1}\right) \otimes \mathcal{F}(2 n+1) .
$$

By induction, we have the map $L_{k}\left(\mathfrak{s o}_{2 n}\right) \hookrightarrow L_{k}\left(\mathfrak{s o}_{2 n+1}\right)$. Then we have an embedding

$$
L_{k+1}\left(\mathfrak{s o}_{2 n}\right) \hookrightarrow L_{k}\left(\mathfrak{s o}_{2 n}\right) \otimes \mathcal{F}(n) \hookrightarrow L_{k}\left(\mathfrak{s o}_{2 n+1}\right) \otimes \mathcal{F}(2 n+1),
$$

where $\mathcal{F}(2 n) \hookrightarrow \mathcal{F}(2 n+1)$ is the isomorphism onto the first $2 n$ copies. Since the image of (5.2) lies in the image of (5.1), it follows that $L_{k+1}\left(\mathfrak{s o}_{2 n}\right)$ embeds in $L_{k+1}\left(\mathfrak{s o}_{2 n+1}\right)$.

This has the following immediate consequence.

Corollary 5.2. For $n \geq 2, m \geq 0$, and $k=-(2 n-2)+\frac{1}{2}(2 n+2 m-1)$, we have an isomorphism

$$
\operatorname{Com}\left(L_{k}\left(\mathfrak{s o}_{2 n}\right), L_{k}\left(\mathfrak{s o}_{2 n+1}\right)\right)^{\mathbb{Z}_{2}} \cong \mathcal{W}_{\ell}\left(\mathfrak{s o}_{2 m+1}\right), \quad \ell=-(2 m-1)+\frac{2 m+2 n-1}{2 m+2 n+1} .
$$

In particular, $\operatorname{Com}\left(L_{k}\left(\mathfrak{s o}_{2 n}\right), L_{k}\left(\mathfrak{s o}_{2 n+1}\right)\right)^{\mathbb{Z}_{2}}$ is strongly rational.

Proof. This follows from Theorem 4.2 together with the fact that $\operatorname{Com}\left(L_{k}\left(\mathfrak{s o}_{2 n}\right), L_{k}\left(\mathfrak{s o}_{2 n+1}\right)\right)^{\mathbb{Z}_{2}}$ is simple, and the map $\mathcal{D}^{k}(n) \rightarrow \operatorname{Com}\left(L_{k}\left(\mathfrak{s o}_{2 n}\right), L_{k}\left(\mathfrak{s o}_{2 n+1}\right)\right)^{\mathbb{Z}_{2}}$ is surjective.

Recall that the category of ordinary modules of an affine vertex algebra at admissible level is semisimple [ArIII] and a vertex tensor category [CHY]. Conjecturally, this category is fusion [CHY] and this has been proven for simply-laced Lie algebras [C]. For type $\mathfrak{s o}_{2 n+1}$ and level $k=-(2 n-2)+\frac{1}{2}(2 n+2 m-1)$ this conjecture is also true. First, $\operatorname{Com}\left(L_{k}\left(\mathfrak{s o}_{2 n}\right), L_{k}\left(\mathfrak{s o}_{2 n+1}\right)\right)$ is a simple current extension, call it $\mathcal{V}_{\ell}\left(\mathfrak{s o}_{2 m+1}\right)$, of $\mathcal{W}_{\ell}\left(\mathfrak{s o}_{2 m+1}\right)$ and thus rational as well [Li]. It follows that $L_{k}\left(\mathfrak{s o}_{2 n+1}\right)$ is a simple $\mathbb{Z}$-graded extension of $L_{k}\left(\mathfrak{s o}_{2 n}\right) \otimes \mathcal{V}_{\ell}\left(\mathfrak{s o}_{2 m+1}\right)$ in a rigid vertex tensor category $\mathcal{C}$ of $L_{k}\left(\mathfrak{s o}_{2 n}\right) \otimes \mathcal{V}_{\ell}\left(\mathfrak{s o}_{2 m+1}\right)$ modules, namely the Deligne product of the categories of ordinary $L_{k}\left(\mathfrak{s o}_{2 n}\right)$-modules and $\mathcal{V}_{\ell}\left(\mathfrak{s o}_{2 m+1}\right)$-modules. Every ordinary module for $L_{k}\left(\mathfrak{s o}_{2 n+1}\right)$ must be an object in this category $\mathcal{C}$. This means that as a braided tensor category the category of ordinary modules of $L_{k}\left(\mathfrak{s o}_{2 n+1}\right)$ is equivalent to the category of local modules for $L_{k}\left(\mathfrak{s o}_{2 n+1}\right)$ viewed as an algebra object in $\mathcal{C}$ [HKL, CKMI]. All assumptions of Theorem 5.12 of [CKMII] are satisfied (with $U=\mathcal{V}_{\ell}\left(\mathfrak{s o}_{2 m+1}\right)$ and $V=L_{k}\left(\mathfrak{s o}_{2 n}\right)$ ) and so

Corollary 5.3. The category of ordinary modules of $L_{k}\left(\mathfrak{s o}_{2 n+1}\right)$ at level $k=-(2 n-2)+\frac{1}{2}(2 n+$ $2 m-1)$ is fusion. 


\section{REFERENCES}

[ArI] T. Arakawa, Associated varieties of modules over Kac-Moody algebras and $C_{2}$-cofiniteness of $\mathcal{W}$-algebras, Int. Math. Res. Not. (2015) Vol. 2015 11605-11666.

[ArII] T. Arakawa, Rationality of $\mathcal{W}$-algebras: principal nilpotent cases, Ann. Math. 182 (2015), no. 2, 565-694.

[ArIII] T. Arakawa, Rationality of admissible affine vertex algebras in the category $\mathcal{O}$, Duke Math. J. 165 (2016) no.1, 67-93

[Ad] D Adamovic, Rationality of Neveu-Schwarz vertex operator superalgebras, Int. Math. Res. Not., 1997:865-874, 1997.

[AKMPP] D. Adamović, V. G. Kac, P. Möseneder Frajria, P. Papi and O. Perše, Finite vs. infinite decompositions in conformal embeddings, Comm. Math. Phys. 348 (2016) no. 2, 445-473.

[ACL] T. Arakawa, T. Creutzig, and A. Linshaw, W-algebras as coset vertex algebras, Invent. Math. 218 (2019), no. 1, 145-195.

[ACKL] T. Arakawa, T. Creutzig, K. Kawasetsu, and A. Linshaw, Orbifolds and cosets of minimal $\mathcal{W}$-algebras, Comm. Math. Phys. 355, No. 1 (2017), 339-372.

[ALY] T. Arakawa, C. H. Lam, and H. Yamada, Parafermion vertex operator algebras and $\mathcal{W}$-algebras, Trans. Amer. Math. Soc. 371 (2019), no. 6, 4277-4301.

[C] T. Creutzig, Fusion categories for affine vertex algebras at admissible levels, Selecta Math. (N.S.) 25 (2019), no. 2, Paper No. 27, 21 pp.

[CGN] T. Creutzig, N. Genra and S. Nakatsuka, Duality of subregular W-algebras and principal Wsuperalgebras, Adv. Math. 383 (2021), 107685, 52 pp.

[CHR] T. Creutzig, Y. Hikida and P. B. Rønne, $N=1$ supersymmetric higher spin holography on AdS ${ }_{3}$, JHEP 02 (2013), 019.

[CHY] T. Creutzig, Y. Z. Huang and J. Yang, Braided tensor categories of admissible modules for affine Lie algebras, Comm. Math. Phys. 362 (2018) no.3, 827-854.

[CKMI] T. Creutzig, S. Kanade and R. McRae, Tensor categories for vertex operator superalgebra extensions, arXiv:1705.05017, to appear in Memoirs AMS.

[CKMII] T. Creutzig, S. Kanade and R. McRae, Glueing vertex algebras, arXiv:1906.00119.

[CLI] T. Creutzig and A. Linshaw, The super $\mathcal{W}_{1+\infty}$ algebra with integral central charge, Trans. Amer. Math. Soc. 367 (2015), no. 8, 5521-5551.

[CLII] T. Creutzig and A. Linshaw, Cosets of affine vertex algebras inside larger structures, J. Algebra 517 (2019) 396-438.

[CLIII] T. Creutzig and A. Linshaw, Trialities of $\mathcal{W}$-algebras, arXiv:2005.10234.

[CV] C. Candu and C. Vollenweider, The $\mathcal{N}=1$ algebra $\mathcal{W}_{\infty}[\mu]$ and its truncations, JHEP 11 (2013), 032.

[DSK] A. De Sole and V. Kac, Freely generated vertex algebras and non-linear Lie conformal algebras, Comm. Math. Phys. 254 (2005), no. 3, 659-694.

[DLY] C. Dong, C. Lam, and H. Yamada, $\mathcal{W}$-algebras related to parafermion algebras, J. Algebra 322 (2009), no. 7, 2366-2403.

[DLM] C. Dong, H. Li, and G. Mason, Compact automorphism groups of vertex operator algebras, Internat. Math. Res. Notices 1996, no. 18, 913-921.

[GL] N. Genra and A. Linshaw, Ito's conjecture and the coset realization of $\mathcal{W}^{k}(s l(3 \mid 2))$, arXiv:1901.02397, to appear in RIMS Kokyuroku Bessatsu.

[HKL] Y. Z. Huang, A. Kirillov and J. Lepowsky, Braided tensor categories and extensions of vertex operator algebras, Comm. Math. Phys. 337 (2015) no.3, 1143-1159.

[I] K. Ito, Quantum Hamiltonian reduction and $N=2$ coset models, Phys. Lett. B 259 (1991) 73-78.

[KW] V. G. Kac and M. Wakimoto, Branching functions for winding subalgebras and tensor products, Acta Appl. Math. 21(1-2): 3-39, 1990.

[KL] S. Kanade and A. Linshaw, Universal two-parameter even spin $\mathcal{W}_{\infty}$-algebra, Adv. Math. 355 (2019), 106774, 58pp.

[Li] H. Li, Extension of vertex operator algebras by a self-dual simple module, J. Algebra 187 (1997) 236-267.

[LI] A. Linshaw, Invariant theory and the Heisenberg vertex algebra, Int. Math. Res. Notices, 17 (2012), 4014-4050.

[LII] A. Linshaw, Invariant subalgebras of affine vertex algebras, Adv. Math. 234 (2013), 61-84.

[LIII] A. Linshaw, Universal two-parameter $\mathcal{W}_{\infty}$-algebra and vertex algebras of type $\mathcal{W}(2,3, \ldots, N)$, Compos. Math. 157 (2021), no. 1, 12-82. 
[W] H. Weyl, The Classical Groups: Their Invariants and Representations, Princeton University Press, 1946.

UNIVERSITY OF AlBERTA

Email address: creutzig@ualberta.ca

UNIVERSITY OF DENVER

Email address: vladimir .kovalchuk@du.edu

UNIVERSITY OF DENVER

Email address: andrew.1inshaw@du .edu 